This item was submitted to Loughborough's Research Repository by the author.

Items in Figshare are protected by copyright, with all rights reserved, unless otherwise indicated.

\title{
Dishing on dinner: a life course approach to understanding the family meal context among families with preschoolers
}

PLEASE CITE THE PUBLISHED VERSION

https://doi.org/10.1017/s1368980020001779

\section{PUBLISHER}

Cambridge University Press (CUP) on behalf of The Nutrition Society

\section{VERSION}

AM (Accepted Manuscript)

\section{PUBLISHER STATEMENT}

This article has been published in a revised form in Public Health Nutrition https://doi.org/10.1017/s1368980020001779. This version is published under a Creative Commons CC-BYNC-ND. No commercial re-distribution or re-use allowed. Derivative works cannot be distributed. ( The authors.

\section{LICENCE}

CC BY-NC-ND 4.0

\section{REPOSITORY RECORD}

Walton, Kathryn, Andrea Breen, Julia Gruson-Wood, Kira Jewell, Emma Haycraft, and Jess Haines. 2020. "Dishing on Dinner: A Life Course Approach to Understanding the Family Meal Context Among Families with Preschoolers". Loughborough University. https://hdl.handle.net/2134/12716789.v1. 


\section{Dishing on Dinner: A life course approach to understanding the family meal context among families with preschoolers}

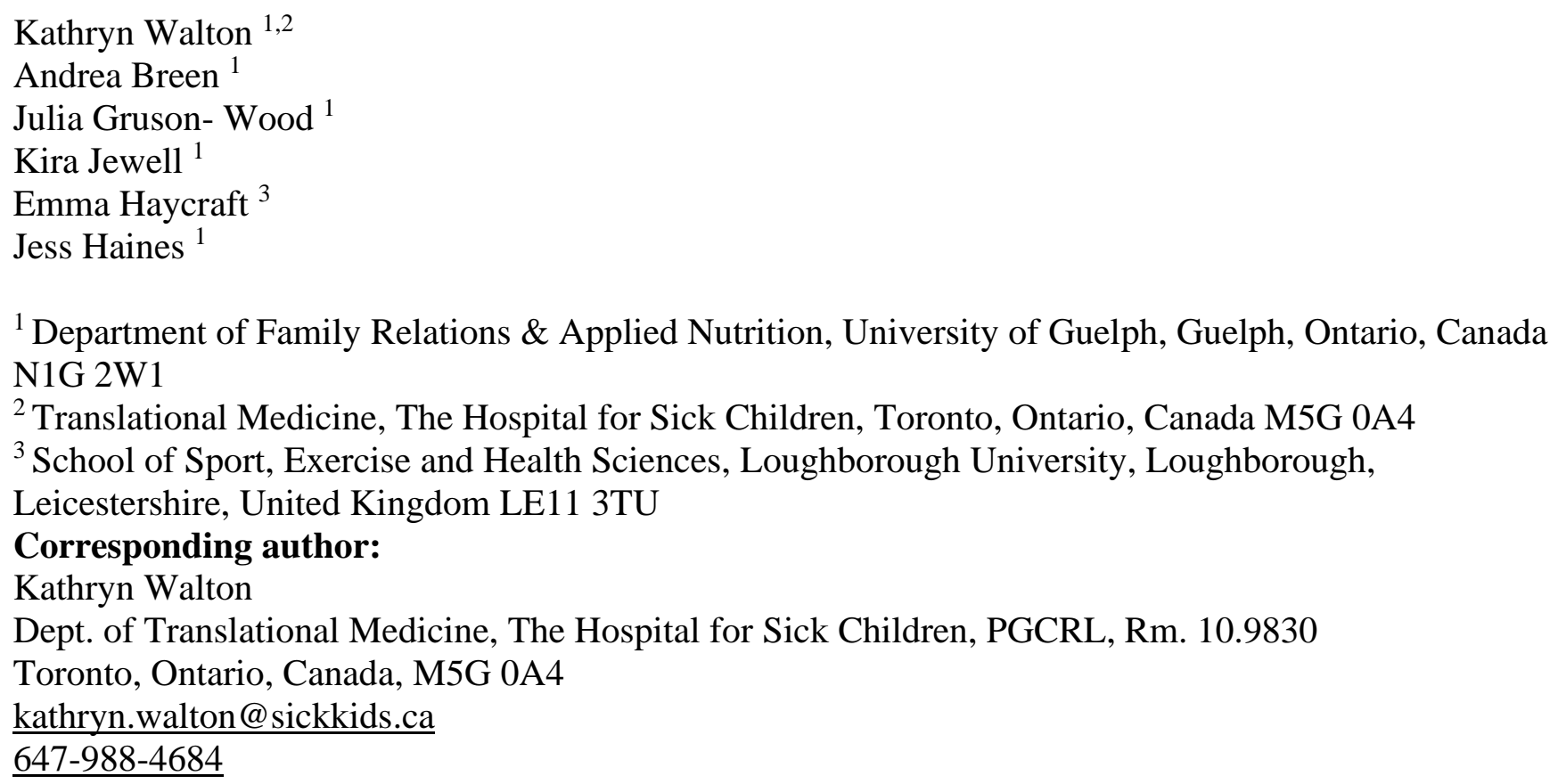

Short title: Dishing on dinner with parents of preschoolers

Conflict of Interest: The authors declare that they have no competing interests.

Funding: Funding provided by the Canadian Foundation for Dietetic Research (CFDR)

Ethical Standards Disclosure: The study was conducted according to the guidelines laid down by the Declaration of Helsinki and all study protocols were approved by the University of Guelph Research Ethics Board (REB\# OC14033). Written informed consent was obtained from all participants.

Authorship: KW, EH \& JH obtained study funding and conceptualized the study design. KW conducted study interviews and drafted the manuscript. $\mathrm{KW}, \mathrm{AB}, \mathrm{JGW}, \mathrm{KJ} \& \mathrm{JH}$ analyzed the data. $\mathrm{AB} \& \mathrm{JGW}$ provided expertise in qualitative methods. All authors interpreted results and reviewed and approved the final manuscript.

Acknowledgements: The authors would like to thank the families who participated in FaMOS and that generously welcomed us into their homes to allow us to learn from their mealtimes. The authors would also like to thank the anonymous reviewers' whose constructive comments significantly improved this manuscript.

Published in Public Health Nutrition 
44 Dishing on Dinner: A life course approach to understanding the family meal context among 45 families with preschoolers

Short title: Dishing on dinner with parents of preschoolers

benefits, family meal participation typically declines as children age. This study utilizes life course theory to explore parents' perceptions of family meals in order to understand how parents' past experiences with family meals (in childhood and earlier in adulthood) influence their current beliefs and practices regarding mealtimes with their own children.

Design: Semi-structured qualitative interviews.

Setting: In-person interviews were conducted in participants' homes.

Participants: Twenty families (21 mothers; 15 fathers) with a child between the ages of 18 months and 5 years.

Results: Thematic analysis revealed that families seemed to primarily approach mealtimes from one of three overarching orientations: meals for 1) Togetherness, 2) Nutrition Messaging or 3) Necessity. These orientations were informed by parents' own mealtime experiences and significant life transitions (e.g., parenthood). The current family meal context, including the messages parents shared with their children during mealtimes and the challenges experienced with mealtimes, characterized the orientations and families' approaches to mealtimes.

Conclusions: Parents' own early life experiences and significant life transitions influence why families eat meals together and have important implications for the intergenerational transmission of mealtime practices. Results may help to inform the content and timing of intervention strategies to support the continuation of frequent family meals beyond the preschool years.

WORD COUNT: 217 
74

75

76

77

78

\section{INTRODUCTION}

Family meals promote healthy eating and wellbeing among both children and adolescents ${ }^{(1)}$. Those who frequently share meals with family members have healthier dietary intakes ${ }^{(1-7)}$ and a lower prevalence of disordered eating ${ }^{(8-11)}$ in comparison to children and adolescents who do not have regular family meals. The benefits extend beyond nutrition, as frequent family meals are associated with lower levels of substance abuse ${ }^{(9,12,13)}$, depressive symptoms ${ }^{(12,14)}$ and improved academic outcomes ${ }^{(12,15,16)}$. However, the frequency of shared meals typically declines linearly beyond the preschool years ${ }^{(17,18)}$. Despite this decline, to date, few family meal interventions have been tested ${ }^{(19)}$. Of the ten existing interventions $^{(20-29)}$, only six present statistically significant intervention effects specific to family meal frequency ${ }^{(20-24,29)}$, the majority of which target adolescents ${ }^{(20,21,23,24)}$ or were tested among a small sample of families ${ }^{(23,24)}$.

Given the equivocal findings of the small body of existing research, many opportunities exist for the development of new approaches to increase family mealtimes and, by extension, improve child health and wellbeing. Findings from the HOME Plus family meal intervention developed by Fulkerson and colleagues ${ }^{(26)}$, suggest that family meal interventions may be more efficacious in impacting healthrelated outcomes such as weight status among younger, pre-pubescent children. The preschool years may be a particularly important window of opportunity as research suggests that health-related behaviours are established early in life and track into adolescence and adulthood ${ }^{(30-33)}$. Thus, to guide the development of effective family meal interventions that sustain frequent family meals beyond the preschool years, we need to understand the context of family meals among families with preschoolers and what motivates or influences parents to establish family meal routines.

Beyond the well-known barriers to family meals, including a lack of time, conflicting schedules and picky eaters ${ }^{(34-36)}$, recent nutrition research highlights the importance of intergenerational influences on family meal routines ${ }^{(37-42)}$. This small body of literature emphasizes that both positive and 
100

101

102

103

104

105

106

107

108

109

110

111

112

114

115

116

117

118

119

120

121

122

123

124

negative childhood mealtime experiences influence how and whether parents engage in mealtimes with their own families ${ }^{(38,39,41)}$. For example, Loth and colleagues ${ }^{(41)}$ described from a nutrition perspective, how parents who participated in frequent family meals from adolescence to adulthood experienced more positive early-life experiences and supports (related to cooking skills, family togetherness and relationships), as compared to those who had inconsistent family meal patterns across time.

Social scientists have long examined eating and family meal practices from a variety of disciplinary perspectives. For example, sociologists have been concerned with understanding how social locations and roles are formed through, and shaped by, eating rituals ${ }^{(43,44)}$. In the context of the family, eating practices have been shown to organize family life and structure the family as a social institution $^{(45,46)}$. Family meals are significant biographical and cultural occasions woven into the fabric of our self-understanding and kinship ties. By bridging the gap between social science and nutrition education research, we are able to interpret the act of eating family dinners as both a "meaningful social practice"(47) and a clinically beneficial activity linked to improved health outcomes.

We aim to extend this previous research by understanding the context in which families with preschoolers participate in family meals and use life course theory ${ }^{(48,49)}$ as a framework from which to understand this phenomenon. Life course theory is an integrative approach to understanding the lives of people over time, emphasizing both personal agency in constructing one's life and the constraints of socio-cultural and historical contexts ${ }^{(48,49)}$. Life course theory emphasizes "linked lives"; the notion that our lives are lived interdependently with others in our family, community and cultural contexts ${ }^{(49)}$. It posits that early life experiences and critical life transitions, such as the transition to parenthood, influence future behaviours ${ }^{(48-50)}$. Employing life course theory to understand the phenomenon of family mealtimes may be particularly useful for enhancing intervention effectiveness ${ }^{(50)}$. Life course theory would suggest that major life transitions and the emergence of new social roles may impact family dietary habits and routines more significantly than incremental or gradual changes in responsibility ${ }^{(48,50)}$. Thus, intervention effects may be stronger when interventions are timed to 
125

126

127

128

129

130

coincide with life transitions and developmental periods that observational studies have associated with an increased interest or likelihood of behaviour change ${ }^{(50)}$.

By approaching families with young children using life course theory, we aim to elucidate how the world of memories, feelings, resources, practices and actions that comprised the eating practices of parents in their childhood years influence the relational dynamics and experiences of their current family meals. Considering the variations with which families' think about and approach family meals is a method for learning how to maintain family mealtime practices over time. The results will help inform whom to target, as well as the timing and content of family meal interventions aimed at helping families sustain frequent family meal participation beyond the preschool years.

\section{METHODS}

\section{Sample and Study Design}

This study was a qualitative sub-study of the (study blinded for peer review), which aimed to explore mealtimes and food parenting practices among 77 families with preschool-aged children ${ }^{(51)}$. Families were eligible to participate in (study blinded for peer review) if: 1) they had a child aged 18 months-5 years, 2) it was typical for the family to eat together, and 3) parents could speak and understand English. A sub-set of 21 families were invited to participate in this qualitative sub-study via email using purposeful, maximum-variation sampling ${ }^{(52,53)}$. Specifically, we purposefully aimed to recruit families that would provide a broad range of family experiences and mealtime perspectives including both single-and dual-headed families and ethnically diverse families. Recruitment ended when we reached theoretical saturation ${ }^{(54,55)}$.

Parents were asked to participate in an in-home, semi-structured interview (guide developed by the authors; Table 1). Interviews lasted approximately 45 minutes. In dual-headed families, both parents were invited to participate in a joint interview and all questions were posed to each parent individually. Following the interview, we sent notes outlining parents' responses to each question to 
151 allow for clarification or additional thoughts. We transcribed interviews verbatim and noted

152 clarifications derived from the member-checking process. We collected demographic information

153 including family structure, mothers' and fathers' ethnicity and educational attainment (university

154 education vs. less than university education), child gender, household income and family dinner

155 frequency as part of (blinded for peer review). Household income was dichotomized using the 2016

156 Canadian low-income cut-offs, which consider total household income before tax and account for

157 population of residence and family size ${ }^{(56)}$. We defined family dinner frequency as the number of times

158 family members eat dinner or supper together; every day (7 days a week), most days (4-6 days a week),

159 a few days (1-3 days a week) or never/rarely (less than one day a week).

\section{Data Analysis}

There were three distinct phases to our qualitative analysis, which followed an inductive

162 approach to thematic analysis ${ }^{(57-60)}$. First, we immersed ourselves in the data by reviewing each

163 transcript, noting emerging ideas and patterns. Two subsequent audio-recorded meetings were

164 conducted to discuss and create initial codes; detailed notes were recorded to document our discussion

165 and decisions ${ }^{(61)}$. In these discussions, we found that our participants' orientation to family meals was

166 governed by one of the following three orientations: meals for 1) Togetherness, 2) Nutrition

167 Messaging, and 3) Necessity. Importantly, the mealtime orientations describe the family-level approach

168 to mealtimes. While parents tended to align themselves primarily with one of the three orientations we

169 identified, they often incorporated the other two orientations into their responses in a complex manner.

170 Individual parent perspectives among dual-headed families did exist and in cases where parents

171 described discordance in their perspectives and experiences, the parents' discussion and interaction

172 during the interview still situated the family unit towards an over-arching mealtime orientation. We

173 began conducting initial analysis while data collection was occurring and concluded data collection

174 when no new ideas were generated beyond what had been provided in earlier interviews; this occurred

175 after 20 families $^{(54)}$. Next, two analysts (initials blinded for peer review) independently coded the 
176 transcripts to compare the mealtime orientations. Segments of data (codes developed during phase 1)

177 were coded using qualitative software (NVivo 11; QSR International Pty Ltd., Melbourne, Australia).

178 Inter-rater reliability calculated by the software revealed a high level of agreement (94\%) between the

179 two analysts; the two resolved any coding discrepancies ${ }^{(60)}$. Finally, we met to review the coding and

180 collate themes ${ }^{(60)}$. We developed four main themes and reviewed them against the data to ensure

181 fidelity throughout the dataset ${ }^{(60)}$. We organized our themes hierarchically with 'mealtime orientation'

182 serving as the overarching framework to understand families' approaches to mealtimes over the life

183 course. The first two themes relate to parents' historical experiences with family meals: 1) early-life

184 meals matter, and 2) stability and change. The remaining themes relate to current meals: 3) mealtime

185 messages and 4) mealtime challenges.

186

\section{RESULTS}

Twenty-one families were invited to participate and twenty families (21 mothers, 15 fathers)

agreed. In two of the dual-headed families one parent was unable to participate due to work conflicts; thus, in 16 families, both parents were interviewed together and in 4 families, the mother was interviewed alone. Table 2 displays demographics of participating families. We identified that families

192 fell predominantly into one of three overarching mealtime orientations (see Table 3), which shape families' approach to meals: 1) Togetherness ( $n=7), 2)$ Nutrition Messaging (n=8), and 3) Necessity $(n=5)$. Briefly, parents in the 'Meals for Togetherness' orientation described that the focus of the family meal is to bring family members together and to connect socially. These parents discussed how social interaction during the meal is more important than the food provided. Parents in the 'Meals for Nutrition Messaging' orientation described that the focus of the family meal is to support healthful eating and discussed how eating together at mealtimes is driven by a desire to ensure their child eats well. Mealtimes are also an opportunity to teach their child about nutrition and portion sizes. Finally, 
201 parenting and that their mealtimes are very functional. These parents described how meals ensure their

202 child eats regularly and serve as a time to teach manners. The two families who reported not

203 participating in family meals every day had a 'Nutrition Messaging' orientation. Both single-parent

204 families described a 'Necessity' orientation. The five families that reported low income fell into either

205 the 'Necessity' or 'Togetherness' orientations, suggesting that socioeconomic status can be a critical

206 factor in shaping parents' mealtime orientation.

207

208

209

Parents' Historical Mealtime Experiences

Early-life meals matter

Our findings expand the literature on the life course by demonstrating the complex ways that

212 their early-life family meals. Parents who described approaching meals with a 'Togetherness'

213 orientation emphasized the social aspects of their childhood meals and remembered childhood

214 mealtimes as full of conversation and connection whereby interactions were warm, and family

215 members enjoyed each other's company. For example, "Rob" described how dinner was the

216 centerpiece of his family life: it was the "time of: "this is our family." Dinner was when family

217 members would "catch up" and talk about "any problems." (F10, Togetherness).

218 In contrast to parents with a 'Togetherness' orientation, parents with a 'Nutrition Messaging' or

219 'Necessity' orientation remember a lack of connection or pleasure during their childhood mealtimes,

220 but in different ways. For example, "Ian" described dinnertime as "interrogation time," with the "main

221 goal" being to "get in and out as quickly as possible"(F16, Nutrition), while "Tyler" recollected that his

222 family did not engage in conversation over dinnertime, rather they "just start[ed] digging for food" ( $F 8$,

223 Necessity). While the first description highlights the complicated and negative family dynamics

224 expressed and tied up in Ian's family meals, Tyler's excerpt illuminates a mealtime environment 
225 focused on the pragmatic task of eating. In both orientations, mealtimes were routinely not a place of 226 enjoyment or connection within the family home.

Participants who described a 'Nutrition Messaging' orientation often discussed receiving

messages about weight, body size and body composition during their childhood meals that continue to impact their relationship with food and family mealtimes. For example, "Shannon" described being told by her parents that healthy foods like "spinach" would make her "strong" like "Popeye" (F19, Nutrition). “George" summed up his parents' mealtime communication as "eat everything but why are you so fat?" (F18, Nutrition). Within the 'Nutrition Messaging' orientation, memories of food also seem to have moral qualities which may stem from intergenerational challenges or fixations on body image and dieting. For example, one mother, "Judy" recalled "watching" her mother go on "many fad diets" and learning "a lot of messages" linking "self-control" with "good body type" (F16, Nutrition).

These messages about body image seem to be one of the reasons many of the parents in this orientation approach meals with a lens towards nutrition. This orientation transition is evident in Judy's case as she now works to cultivate a "healthy relationship with food" by talking about "consumption" with her daughters through the lens of "nutrition."

Parents in the other orientations did not report memories of nutrition messaging or dieting.

Notably however, parents in the 'Necessity' orientation described their childhood mealtimes as marked by pressure to consume food as a functional requirement of survival. "Jeff” describes his childhood meals as a time to get fed and move on to other activities: "[Meals were] more just like 'feed the family' instead of trying to eat particularly healthy food.” (F5, Necessity). Similarly, "Kristen,” a mother with a 'Necessity' orientation describes having no memory of her parents "saying anything about healthy or unhealthy eating," it was just: "was there food?" She continued to describe memories of "having cereal with no milk for breakfast" and "eating cheese and salad dressing because that's all there was" $(F 17)$. Kristen explained how she tries to balance out being "a little more inclusive of food groups" with her children while "trying to live within a really strict budget." She described this 
250 balancing act as making it "a little bit more difficult to be health conscious all the time." Here, lack of

251 financial resource created a particular orientation towards food that, along with economic status, has

252 been passed down intergenerationally. The ability to focus on the nutritional value of food is a

253 privilege and the 'Necessity' orientation highlights how socio-economic status may impact the 254 development of mealtime orientations.

Our results demonstrate the importance of early life on the trajectory of mealtime orientations

and routines. Parents who currently approach meals for family connection remembered their childhood

mealtimes in the same positive light and seemed to have a more positive relationship with eating in

relation to their self-development and body image. The others shared elements of disconnection,

stemming from complicated family dynamics, diet culture and the impact of food insecurity.

For most parents, their childhood mealtime orientations tracked as a stable trajectory into

adulthood, providing lifelong context for their eating habits and mealtime experiences. The

'Togetherness' orientation seemed to be the most stable, with almost all parents currently approaching meals from this orientation also describing their childhood meals as a time for family connection.

However, consistent with life course theory, there were major points in life where mealtime

orientations changed $^{(48,50,62,63)}$. For parents whose childhood meal orientations did not track into adult relationships and being immersed in their partner's food culture and 2) the transition to parenthood. Parents reported that these life events created deep, fundamental and lasting changes to their mealtime routines. For example, "Claire" described liking the social aspect of her husband's family mealtimes. She contrasted this with her childhood mealtime experiences which were marked by

272 her father wanting everyone to be "quiet" while eating. (F10, Togetherness). Judy described the "clash 273 of cultures" between her history with family meals and that of her husband. While her own parents 274 offered only "homemade" dinners, her husband grew up on "processed" food. She pronounced: "I won 
275 that battle," to which her husband, Ian responded, "I guess so. There wasn't really [that] sort of 276 messaging about [...] food" (F16, Nutrition). These quotes illustrate how as partners, parents

277 negotiated and were influenced by each other's experiences and orientations towards shared meals. In 278 these examples, both Claire and Ian's childhood mealtime orientations were altered to align with those 279 of their partner. Notably, this was not the case for all dual-headed families like "Vicky" and "Owen's" 280 family, where parents' trajectories remained stable and they "agreed to be different" while mostly 281 defaulting towards one parent's approach (F4, Togetherness). Vicky explains, “well it doesn't work 282 that we have different, um, philosophies. [...] like I said [mealtimes are] family time", while Owen 283 says, "we sit together to get fed" $(F 4)$. This process of negotiating, compromising or disagreeing on 284 approaches to family mealtimes highlights the importance of considering the perspectives of both 285 parents in dual-headed homes and further demonstrates how eating rituals are shaped by social roles, as 286 both a partner and a parent.

While mealtime practices shift throughout the life course, parents still seemed to orient to their 288 childhood mealtime practices — even if as a counterpoint — in defining their current family mealtimes. 289 While family practices may diminish during early adulthood, in the context of major life transitions 290 such as becoming a parent, latent influences such as where meals should be eaten, remerged. For 291 example, "Mia" (F14, Nutrition) described how before having kids she used to eat dinner in front of the 292 TV with her spouse. Having children motivated her to create a space to sit together for meals. 293 Similarly, Mia's husband "Lucas" started having regular family meals for the first time after becoming 294 a father; he describes how as a child he would grab dinner and "go to my room and watch TV by 295 myself and eat like on my bed" (F14). "Jeff" said that when he became a father, he purposefully chose 296 to avoid pressuring his son to eat, which is a pointed counteraction to his childhood. He described 297 "everything” around mealtime being "a power struggle," during his own childhood which resulted in 298 the resolution to never "force" his child to eat when "he says he's not hungry" (F5, Necessity). 
For other families — particularly 'Nutrition Messaging' — the transition to parenthood seemed to

300 negatively impact the mealtime orientation. This transition seemed to be associated with anxiety, to the 301 point at which their orientation towards meals shifted from Togetherness to Nutrition. Mia describes

302 'obsessing' about the food her daughter eats, which contrasts with the positive relationship she had 303 with food as a child. She described her childhood as enabling her the freedom of "consum[ing] as much 304 food" as she "wanted" yet in her adult years she has become "consumed" and "obsessed" with her own, 305 and her daughter's, intake (F14, Nutrition). Judy reflects on how having three kids and coming from a 306 "very long line of body shaming women," has caused her to develop a relationship with food that 307 centers around body image concerns (F16, Nutrition).

To this end, having a child created positive mealtime changes for some, and negative changes

309 for others. Some described a mix of both positive and negative influences on their mealtime routines

310 including starting to have mealtimes but becoming more worried about the types of foods to serve and 311 wondering whether their child was eating enough. Whether the transition to parenthood had positive or 312 negative implications on mealtime orientation seemed to stem from the parents' early-life experiences, 313 with latent expectations or challenges remerging.

Impact of Historical Meal Experiences on Current Family Meal Context: Mealtime Messages

Parents' experiences with family meals throughout life influenced their mealtime orientation 318 which included the food and eating practices that they currently share with their children. The mealtime 319 orientation of the parent participants interviewed served as the contextual foreground for the way they 320 'do' food and family meals. Embedded in parents' orientations are larger family dynamics (See Table $3213)$. 
Consistent with life course theory, we found that not only do mealtime routines shift and change

as the family unit evolves ${ }^{(48,63)}$, but so do the challenges that parents experience when sharing

mealtime messages with their children. All families noted some challenges, however the main

challenges and their impact differed across orientations.

'Togetherness' families reported few challenges in sharing their message of connection but noted that this may change as their child gets older and other activities (e.g. extra-curriculars and parttime jobs) contest family meals. "Nicole" reflected how, as her children age, the family "might not be sitting together at the table" leading to concern about "finding time, to have those really important conversations." (F1).

'Nutrition Messaging' families reported feelings of general frustration towards mealtimes, which challenged their ability to focus on enjoyment during meals. For example, "Alex" described supper as not "always having a positive feeling and stated that she "blame[d] her children for that" (F19), while "Emily" described dinner as "just such a fraught thing..." (F2). Some of these challenges may be related to parents' reports of wanting to control their child's intake during mealtimes. Mia described the importance of providing "good portion control" to her toddler and the push back she received from her partner who "doesn't think" toddlers require this form of regulation (F14). Later in the interview Mia explicitly expressed concern that her daughter might follow her habit of linking food to self-image. She described wanting her daughter to be able to "think about [eating] in a positive way, that reflects a good self-image" and not wanting food to be "so tied to physical appearance" but struggled to model this herself.

Parents with a 'Necessity' orientation talked about wanting mealtimes to provide structure to their child's day and to have their children eat healthy, but also reported difficulties in modeling these healthy behaviours. For example, "Brittany" described making breakfast for her son and then realizing hours later that she had not eaten yet $(F 11)$. Kristen described a desire to be "health conscious" while also choosing fast food in "certain situations" because "it's cheap and fast" (F17). These parents also 
348 reported scheduling changes to be disruptive to their participation in family meals due to busyness with 349 appointments and after school activities as described by "Diane": "[We] grab something on the road 350 because of a lack of time." (F8, Necessity) Interestingly, our quantitative data does not reflect scheduling interruptions among families with a 'Necessity' orientation. It was families with a 'Nutrition Messaging' orientation that reported not participating in family meals every day, highlighting the importance of focusing on the underlying mealtime orientation that leads to frequency. Families may define family meals differently depending on the orientation with which they appreach meals. Given the internal pressure that parents with at 'Autrition Messaging' orientation seem place on themselves, they may not count meats that do not meet certain criteria in quantitative reports of shared meals, despite the benefits they still have for child wellbeing.

Challenges were the most prevalent among families with a 'Nutrition Messaging' or 'Necessity' orientation. Parents with a 'Nutrition Messaging' orientation discussed frustration towards mealtimes stemming from their responsibility of providing healthy meals and desire for control over their children's diets. Parents with a 'Necessity' orientation discussed wanting family meals to provide structure to their children's days, but found it challenging to model because they often prioritize 364 feeding their children over themselves.

\section{DISCUSSION}

Across all mealtime orientations, we found that parents' early-life meal routines and experiences informed the trajectory and context of their current family meals. Similar to our findings, Malhotra and colleges ${ }^{(39)}$ found that mothers' childhood experiences informed their perceptions of the benefits and barriers to family meals with preschoolers. Trofholz ${ }^{(38)}$ found that the lessons mothers 371 learned about family meals from their parents influenced the lessons they shared with their school-aged 372 children. Loth and colleagues ${ }^{(41)}$ recently described the intergenerational transmission of mealtime 
373 routines based on frequency of participation using three different longitudinal trajectories from

374 adolescence to adulthood: maintainers, starters, and inconsistent. Those who were considered

375 maintainers of regular family meals reported many of the same mealtime supports that were discussed

376 by parents with a 'Togetherness' orientation, including positive childhood memories about family

377 meals and having a partner who shared a similar upbringing with family meal participation or who also

378 valued family meals. Our study extends previous research by considering how other significant life

379 changes impact these experiences, and in such a way as to form particular orientations that shape 380 everyday practice.

Using life course theory to frame our exploration of the family mealtime context led to

important insights useful for the development of future interventions aimed at sustaining frequent

family meal participation beyond the preschool years. We determined that, for many parents, childhood

mealtime routines and orientations seemed to track into parenthood, illustrating the importance of

intervening among families with young children to impact the intergenerational transmission of this

routine. However, we also found that major life transitions, e.g., partnership/marriage or parenthood,

appear to impact the family's mealtime routines and orientation ${ }^{(50)}$. For some, life transitions may cause

changes to these practices; dietary routines and behaviours are fluid which signals important windows

of opportunity for establishing family meals ${ }^{(64)}$. Examining parent's mealtime practices and beliefs

from a life course perspective provides a deeper understanding of factors underlying mealtime supports

or barriers. For example, 'Nutrition Messaging' parents described frustration towards mealtimes, but

our use of life course theory provided the understanding that, in some cases, this frustration may stem

from transgenerational struggles with body image and a resultant internal pressure to ensure meals are

healthy. Further, while families leaned towards an overarching mealtime orientation, there was some 
towards shared meals. Future research should explore these mealtime orientations over time to understand how co-parenting with different mealtime philosophies impacts the frequency of family meals and the mealtime environment. It is possible that over the life course, differing orientations may dominate the family's approach to family meals depending on context and life demands at particular points in time.

Our results underscore that grounding in social science theory, specifically life course theory, may help clinicians and practitioners to understand the constellation of parents' beliefs, experiences and practices with respect to mealtimes. This understanding will help to create interventions that are grounded in understanding parents' specific mealtime orientations and provide tailored support.

\section{Implications for Practice and Research}

Like in previous research, the preschoolers in this study participated in frequent family meals ${ }^{(5,}$

18,65-67). Our results extend this by highlighting important variations in the mealtime environments and philosophies among families who participate in frequent family meals. Our results suggest that it is important to understand why families eat together to properly understand the challenges they may face and to provide tailored supports. Interventions should be aimed at sustaining this high frequency of participation throughout childhood and into adolescence. Effective intervention programs may require learning and responding to each family's mealtime orientation. To further explain, we will now turn to a focused discussion of the implications of each mealtime orientation and the specific intervention messages and considerations for healthcare professionals.

\section{Meals for Togetherness}

Almost all participants who embodied this orientation maintained it throughout their lives, despite life transitions. Parents who adopt this orientation appear to focus on the importance of the social aspects of family meals, which sets the context for relaxed mealtimes and reduced anxiety towards their children's intake. While their children are small, they do not seem to struggle with finding time for mealtimes but acknowledge that this may be a challenge in the future. These families can be 
encouraged to continue eating together for family bonding and reminded that their modelling during this time will set their children up for positive psychosocial development ${ }^{(36)}$ and lifelong healthy eating $^{(1,68)}$. To continue having family meals as their children age, these families may benefit from being reminded that any meal counts in terms of providing benefits to their children. Because parents with this orientation described strong intergenerational stability in their meal routines, these families may not need to be the focus of intervention or supports aimed at sustaining family meals over time.

\section{Meals for Nutrition Messaging}

Parents with this orientation tended to describe mealtimes as a source of anxiety and frustration due to their concern about their family's nutritional intake. Our finding that the two families who identified that they do not have family meals every day both identified with a 'Nutrition Messaging' orientation may suggest that this frustration may already impact the frequency with which families within this orientation eat together. Alternatively, given the internal pressure that these parents seem place on themselves, they may not count meals that do not meet certain criteria in quantitative reports of shared meals, despite the benefits they still have for child wellbeing. However, our data cannot determine the direction of this effect and it is also possible that this lower frequency of shared meals is contributing to parents' frustration. This orientation seems to have been particularly vulnerable to increased mealtime anxiety during the transition to parenthood. Healthcare professionals should acknowledge the family's participation in shared meals as an excellent way to promote healthy eating habits. However, given their less favorable perception of and anxiety towards family meals, these families may struggle to continue the practice of eating together as children gain independence; mealtimes may also be fraught with power struggles surrounding eating. Harris and colleagues ${ }^{(69)}$ suggest the importance of identifying families that have concerns early (regardless of the presence of an actual feeding or growth problem), before escalating parental worry during feeding interactions negatively impacts the food parenting practices used (more pressuring tactics, including control) and the resultant mealtime environment. These families may benefit from support to take a more relaxed 
448 approach to family meals that focuses on the social benefits and enjoyment of family meals with a 449 reduced focus specifically on nutrition. Given parents reported desire to control their child's intake 450 during meals, and their challenges with body image, some may benefit from counselling supports for 451 their own food challenges. Meals for Necessity Parents with this orientation describe family meals as necessary to accomplish the task of child feeding. They also noted mealtimes as important for providing structure to their children's day, but noted many challenges associated with the routine. Families with this orientation should be encouraged that mealtimes are an excellent way to create routine and structure to their child's day; research strongly supports the benefits of routines in young children's development ${ }^{(29,70)}$. These families may struggle to continue the tradition of eating together as children age and no longer require supervision during meals, especially among single-headed families where scheduling may be more challenging. Health professionals are encouraged to reiterate to families that these routines and structure are also beneficial for children as they grow up. Families in this orientation reported struggling to find time to eat together and reported that this routine is interrupted when other life responsibilities arise. Health professionals can also help families to problem solve through challenges when they arise and provide supports for making the process easier (i.e. quick and easy recipes, reminders that any meal eaten together counts). Given our results that 'Necessity' families had lower household income, these families will likely benefit from support surrounding meal planning on a budget and, where possible, 467 financial and systematic supports to make healthy food more accessible. The parents with this orientation also acknowledged challenges in modelling healthful eating habits for their children related to a lack of time, resources and in some cases, nutrition knowledge. While positive dietary modelling is beneficial for children ${ }^{(71)}$, before creating an intervention, it is important to understand how these barriers shape parents articulated challenges with modelling healthy eating. While some parents may 472 benefit from dietary counselling to support their own behaviour change, the socio-economic barriers to 
473 parents' healthful eating habits must not be lost from view. Particularly for families that

474 have experienced food insecurity - currently or in the past, the topic of healthy eating may be

475 particularly loaded. Rather than narrowing in on modelling or nutrition education per se, building on

476 parents' current goals and focusing on additional benefits like family bonding while providing supports

477 tailored to individual family challenges will be constructive in supporting healthful shared meals long478 term.

\section{Strengths and Limitations}

By acknowledging nutrition as a socially inflected category, we were able to examine the nutritional practices of family meals in a sociological manner to enhance both clinical nutrition practice and the development of family meal interventions. To our knowledge, this is the first study of this kind within Canada, which is important as family meal and parenting expectations may vary across cultures and countries ${ }^{(64,72)}$. Our study also provides a dual-parent perspective for a more complete understanding of the family meal context. However, there are limitations to note. Families signed up to participate in a study about mealtimes and, as such, may have more interest in mealtimes and food than families that did not sign up. While we found diversity in the mealtime orientations described, the orientations may not extend to those outside the study, to families with older children, or to families that share fewer meals or do not participate in family meals at all. The majority of families participating in this study had fairly high income and educational attainment and the majority of parents identified as 'white'; thus, results may not be generalizable to other populations. However, owing to our purposeful, maximum-variation sampling technique, the families participating in this qualitative sub-study were more ethnically diverse than the full study ${ }^{(51)}$. Future research should explore our mealtime orientations among more diverse populations and longitudinally across the lifespan. 
In this study we explored parents' perceptions and experiences of family meals. Understanding

498 how people make sense of eating and how their relationship with food intertwines lived experiences in

499 family and socio-cultural contexts across the life course provides the knowledge required to create

500 effective interventions geared to sustaining frequent family meals beyond the preschool years.

501 Accordingly, we found participating families to primarily embody one of three overarching mealtime

502 orientations that informed the context of their meals, the messages they share with their children during

503 meals, and the challenges they experienced with mealtimes. Results suggest that understanding why

504 families eat meals together has important implications for the intergenerational transmission of

505 mealtime practices, as well as the intervention strategies that are likely to be beneficial to help families

506 sustain the routine as their children age. While meal orientations often track throughout the life course,

507 they are also fluid and vulnerable to life transitions. Future research should focus on understanding

508 these orientations in more diverse populations and testing their ability to inform practice in a variety of

509 settings including dietetic counselling and intervention planning.

510 WORD COUNT: 5693 
528

529

530

531

532

533

534

535

536

537

538

539

540

541

542

543

544

545

546

547

548

549

550

551

552

553

554

555

556

557

558

559

560

561

562

563

564

565

566

567

568

569

570

571

572

\section{REFERENCES}

1. Hammons AJ \& Fiese BH (2011) Is frequency of shared family meals related to the nutritional health of children and adolescents? Pediatrics 127, e1565-1574.

2. Neumark-Sztainer D, Hannan PJ, Story M et al. (2003) Family meal patterns: associations with sociodemographic characteristics and improved dietary intake among adolescents. J Am Diet Assoc 103, 317-322.

3. Cooke L, Wardle J, Gibson EL et al. (2004) Demographic, familial and trait predictors of fruit and vegetable consumption by pre-school children. Public Health Nutr 7, 295-302.

4. Fitzpatrick E, Edmunds LS Dennison BA (2007) Positive effects of family dinner are undone by television viewing. J Am Diet Assoc 107, 666-671.

5. Wyse R, Campbell E, Nathan N et al. (2011) Associations between characteristics of the home food environment and fruit and vegetable intake in preschool children: a cross-sectional study. BMC Public Health 11, 938.

6. Woodruff SJ \& Hanning RM (2008) A review of family meal influence on adolescents' dietary intake. Can J Diet Pract Res 69, 14-22.

7. Swanson V, Power KG, Crombie IK et al. (2011) Maternal feeding behaviour and young children's dietary quality: a cross-sectional study of socially disadvantaged mothers of twoyear old children using the Theory of Planned Behaviour. Int J Behav Nutr Phys Act 8, 65.

8. Ackard DM \& Neumark-Sztainer D (2001) Family mealtime while growing up: associations with symptoms of bulimia nervosa. Eat Disord 9, 239-249.

9. Fulkerson JA, Story M, Mellin A et al. (2006) Family dinner meal frequency and adolescent development: relationships with developmental assets and high-risk behaviors. J Adolesc Health 39, 337-345.

10. Neumark-Sztainer D, Wall M, Story M et al. (2004) Are family meal patterns associated with disordered eating behaviors among adolescents? J Adolesc Health 35, 350-359.

11. Neumark-Sztainer D, Eisenberg ME, Fulkerson JA et al. (2008) Family meals and disordered eating in adolescents: longitudinal findings from project EAT. Arch Pediatr Adolesc Med 162, 17-22.

12. Eisenberg ME, Olson RE, Neumark-Sztainer D et al. (2004) Correlations between family meals and psychosocial well-being among adolescents. Arch Pediatr Adolesc Med 158, 792-796.

13. Goldfarb SS, Tarver WL, Locher JL et al. (2015) A systematic review of the association between family meals and adolescent risk outcomes. J Adolesc 44, 134-149.

14. Fulkerson JA, Kubik MY, Story M et al. (2009) Are there nutritional and other benefits associated with family meals among at-risk youth? The Journal of adolescent health : official publication of the Society for Adolescent Medicine 45, 389-395.

15. Snow CE \& Beals DE (2006) Mealtime talk that supports literacy development. New Dir Child Adolesc Dev, 51-66.

16. Beals D (2001) Eating and reading: links between family conversations with preschoolers and later language and literacy. In Beginning literacy with language: young children learning at home and school [D Dickinson and P Tabors, editors]. Baltimore, MD: Brookes Publishing.

17. McCullough MB, Robson SM Stark LJ (2016) A Review of the Structural Characteristics of Family Meals with Children in the United States. Advances in Nutrition 7, 627-640.

18. Miller DP, Waldfogel J Han WJ (2012) Family meals and child academic and behavioral outcomes. Child Dev 83, 2104-2120. 
573

574

575

576

577

578

579

580

581

582

583

584

585

586

587

588

589

590

591

592

593

594

595

596

597

598

599

600

601

602

603

604

605

606

607

608

609

610

611

612

613

614

615

616

19. Dwyer L, Oh A, Patrick $\mathrm{H}$ et al. (2015) Promoting family meals: a review of existing interventions and opportunities for future research. Adolesc Health Med Ther 6, 115-131.

20. DeBar LL, Stevens VJ, Perrin N et al. (2012) A primary care-based, multicomponent lifestyle intervention for overweight adolescent females. Pediatrics 129, e611-620.

21. Sepúlveda MJ, Lu C, Sill S et al. (2010) An observational study of an employer intervention for children's healthy weight behaviors. Pediatrics 126, e1153-1160.

22. Johnson DB, Birkett D, Evens $C$ et al. (2006) Promoting family meals in WIC: lessons learned from a statewide initiative. J Nutr Educ Behav 38, 177-182.

23. Rosenkranz RR \& Dzewaltowski DA (2009) Promoting better family meals for girls attending summer programs. J Nutr Educ Behav 41, 65-67.

24. Utter J, Denny S, Farrant B et al. (2019) Feasibility of a Family Meal Intervention to Address Nutrition, Emotional Wellbeing, and Food Insecurity of Families With Adolescents. J Nutr Educ Behav 51, 885-892.

25. Ayala GX, Elder JP, Campbell NR et al. (2010) Longitudinal intervention effects on parenting of the Aventuras para Niños study. Am J Prev Med 38, 154-162.

26. Fulkerson JA, Friend S, Flattum C et al. (2015) Promoting healthful family meals to prevent obesity: HOME Plus, a randomized controlled trial. Int J Behav Nutr Phys Act 12, 154.

27. Fulkerson JA, Rydell S, Kubik MY et al. (2010) Healthy Home Offerings via the Mealtime Environment (HOME): feasibility, acceptability, and outcomes of a pilot study. Obesity (Silver Spring) 18 Suppl 1, S69-74.

28. White AA, Colby SE, Franzen-Castle L et al. (2019) The iCook 4-H Study: An Intervention and Dissemination Test of a Youth/Adult Out-of-School Program. J Nutr Educ Behav 51, S2-S20.

29. Haines J, McDonald J, O'Brien A et al. (2013) Healthy Habits, Happy Homes: randomized trial to improve household routines for obesity prevention among preschool-aged children. JAMA Pediatr 167, 1072-1079.

30. Savage JS, Fisher JO Birch LL (2007) Parental influence on eating behavior: conception to adolescence. J Law Med Ethics 35, 22-34.

31. Birch L \& Fisher J (1998) Development of eating behaviors among children and adolescents. Pediatrics 101, 539-549.

32. Birch L (1999) Development of food preferences. Annu Rev Nutr 19, 41-62.

33. Skinner JD, Carruth BR, Wendy B et al. (2002) Children's food preferences: a longitudinal analysis. J Am Diet Assoc 102, 1638-1647.

34. Martin-Biggers J, Hongu N, Worobey J et al. (2013) Family meal behaviors and cognitions among parents of preschoolers. FASEB $J 27$.

35. Quick BL, Fiese BH, Anderson B et al. (2011) A formative evaluation of shared family mealtime for parents of toddlers and young children. Health Commun 26, 656-666.

36. Martin-Biggers J, Spaccarotella K, Berhaupt-Glickstein A et al. (2014) Come and get it! A discussion of family mealtime literature and factors affecting obesity risk. Adv Nutr 5, 235-247.

37. Watts A, Berge JM, Loth K et al. (2018) The Transmission of Family Food and Mealtime Practices From Adolescence to Adulthood: Longitudinal Findings From Project EAT-IV. J Nutr Educ Behav 50, 141-147.e141.

38. Trofholz AC, Thao MS, Donley M et al. (2018) Family meals then and now: A qualitative investigation of intergenerational transmission of family meal practices in a racially/ethnically diverse and immigrant population. Appetite 121, 163-172. 
617

618

619

620

621

622

623

624

625

626

627

628

629

630

631

632

633

634

635

636

637

638

639

640

641

642

643

644

645

646

647

648

649

650

651

652

653

654

655

656

657

658

659

660

661

39. Malhotra K, Herman AN, Wright G et al. (2013) Perceived benefits and challenges for lowincome mothers of having family meals with preschool-aged children: childhood memories matter. J Acad Nutr Diet 113, 1484-1493.

40. Berge JM, Miller J, Watts A et al. (2018) Intergenerational transmission of family meal patterns from adolescence to parenthood: longitudinal associations with parents' dietary intake, weight-related behaviours and psychosocial well-being. Public Health Nutr 21, 299-308.

41. Loth KA, Uy MJA, Winkler MR et al. (2019) The intergenerational transmission of family meal practices: a mixed-methods study of parents of young children. Public Health Nutr 22, 12691280.

42. Friend S, Fulkerson JA, Neumark-Sztainer D et al. (2015) Comparing childhood meal frequency to current meal frequency, routines, and expectations among parents. J Fam Psychol 29, 136140.

43. Ashley B, Hallows J, Jones S et al. (2004) Food and Cultural Studies. London \& New York: Routledge.

44. Cairns K \& Johnson J (2015) Food and Femininity. New York, NY: Bloomsbury Academic.

45. DeVault M (1991) Feeding the Family: The Social Organization of Caring as Gendered Work, Women in Culture and Society. Chicago and London: University of Chicago Press.

46. Ristovski-Slijepcevic S, Chapman GE Beagan BL (2010) Being a 'good mother': Dietary governmentality in the family food practices of three ethnocultural groups in Canada. Health (London) 14, 467-483.

47. Crawford R (2006) Health as a meaningful social practice. Health (London) 10, 401-420.

48. Elder J (1995) The life course paradigm: Social changes and individual development. In Examining Lives in Context, pp. 101-139 [P Moen, GJ Elder and K Luscher, editors]: American Psychological Association.

49. Elder GJ, Johnson M, Crosnoe et al. (2003) The emergence and development of life course theory. In Handbook of the life course, pp. 3-19 [J Mortimer and M Shanahan, editors]. New York: Kluwer.

50. Wethington E \& Johnson-Askew WL (2009) Contributions of the life course perspective to research on food decision making. Ann Behav Med 38 Suppl 1, S74-80.

51. Walton K, Haycraft E, Jewell K et al. (2019) The Family Mealtime Observation Study (FaMOS): Exploring the Role of Family Functioning in the Association between Mothers' and Fathers' Food Parenting Practices and Children's Nutrition Risk. Nutrients 11.

52. Patton M (1990) Designing Qualitative Studies. In Qualitative evaluation and research methods, pp. 169-186. Beverly Hills, CA: Sage.

53. Palinkas LA, Horwitz SM, Green CA et al. (2015) Purposeful Sampling for Qualitative Data Collection and Analysis in Mixed Method Implementation Research. Adm Policy Ment Health 42, 533-544.

54. Saunders B, Sim J, Kingstone T et al. (2017) Saturation in qualitative research: exploring its conceptualization and operationalization. In Quality \& Qunatity, pp. 1-15.

55. Sobal J (2001) Sample extensiveness in qualitative nutrition education research. J Nutr Educ 33, 184-192.

56. Statistics Canada (2006) Low Income Cut-offs for 2006 and Low Income Measures for 2005. Low Income Research Paper Series.

57. Braun V \& Clarke V (2006) Using thematic analysis in psychology. Qualitative Research in Psychology 3, 77-101. 
662

663

664

665

666

667

668

669

670

671

672

673

674

675

676

677

678

679

680

681

682

683

684

685

686

687

688

689

690

691

692

693

694

695

696

697

698

699

700

701

702

703

704

705

706

707

708

58. Vaismoradi M, Turunen $\mathrm{H}$ Bondas $\mathrm{T}$ (2013) Content analysis and thematic analysis: Implications for conducting a qualitative descriptive study. Nurs Health Sci 15, 398-405.

59. Hsieh HF \& Shannon SE (2005) Three approaches to qualitative content analysis. Qual Health Res 15, 1277-1288.

60. Braun V \& Clarke V (2013) Successful Qualitative Research: a practical guide for beginners. Thousand Oaks, CA: Sage.

61. Mays N \& Pope C (1995) Rigour and qualitative research. BMJ 311, 109-112.

62. Setterstein R (1999) Lives in time and Place: The Problems and Promises of Developmental Science. Amityville: Baywood.

63. Elder J (2003) The emergence and development of life course theory. In Handbook of the Life Course, pp. 3-19 [E Mortimer and M Shanahan, editors]. New Yrok: Kluwer.

64. Moura AF \& Aschemann-Witzel J (2020) A downturn or a window of opportunity? How Danish and French parents perceive changes in healthy eating in the transition to parenthood. Appetite 150, 104658.

65. Litterbach EV, Campbell KJ Spence AC (2017) Family meals with young children: an online study of family mealtime characteristics, among Australian families with children aged six months to six years. BMC Public Health 17, 111.

66. Berge JM, Truesdale KP, Sherwood NE et al. (2017) Beyond the dinner table: who's having breakfast, lunch and dinner family meals and which meals are associated with better diet quality and BMI in pre-school children? Public Health Nutr 20, 3275-3284.

67. Le Heuzey M \& Turberg- Romain C (2015) Nutri bébé 2013 Study Part 3/Behaviours of mothers and young children during feeding. Archives de Pediatrie 22.

68. Patrick H \& Nicklas TA (2005) A review of family and social determinants of children's eating patterns and diet quality. J Am Coll Nutr 24, 83-92.

69. Harris HA, Ria-Searle B, Jansen E et al. (2018) What's the fuss about? Parent presentations of fussy eating to a parenting support helpline. Public Health Nutr 21, 1520-1528.

70. Spagnola M \& Fiese B (2007) Family Routines and Rituals: A Context for Development in the Lives of Young Children. Infants \& Young Children 20, 284-299.

71. Pearson N, Biddle SJ Gorely T (2009) Family correlates of fruit and vegetable consumption in children and adolescents: a systematic review. Public Health Nutr 12, 267-283.

72. Bornstein MH (2012) Cultural Approaches to Parenting. Parent Science Practice 12, 212-221. 
709

710

Table 1: Semi-Structured Interview Guide Exploring Parent Feeding Practices and Family Meal Experiences

1. What do you feel works well with regards to your family's meal routine?

2. What does not work as well?

3. Think back to your own childhood. What do you remember about mealtimes? [Probe with the following]: Can you remember a specific meal that you shared with your family? Please describe it is as much detail as you can. Who was there, what do you remember, what makes it memorable? Was this meal a typical experience in your house?

4. How would you describe your relationship to food and eating? Has this changed over time?

5. What kind of messages about food and eating did you get from your parents growing up?

6. Besides your family growing up, what else has influenced your relationship with food and the way you approach eating in your own life?

7. What is the main message you want your child to learn about food and eating? What messages do you hope your child will get from you?

8. How successful do you feel in this? [Prompt] If parent(s) do not feel successful, ask: What do you think would help you experience more success in this? 
738

739

Table 2: Demographics of Participating Families

Target Child $(n=20)$ and Parent Characteristics $(n=36 ; 21$ mothers*, 15 fathers)

Gender of Target Child, n (\%)

Male

Age of target child (years), mean (SD)

Maternal Ethnicity, n (\%)

White

Paternal Ethnicity, n (\%)

White

Maternal Educational Attainment, n (\%)

University education or higher

Paternal Educational Attainment, n (\%)

University education or higher

Family-level Characteristics $(\mathbf{n}=\mathbf{2 0})$

Dual-Headed Home**, n (\%)

$15 / 21(71)$

$10 / 15(66)$

\# of children in the home, mean (SD)

Low Income

Yearly Income below population and family-size adjusted cut-offs

Family Dinner Frequency

Every day (7 days/ week)

$18(90)$

Most days (4-6 days/week)

$2(10)$

*One same-sex couple

**Among the 18 dual-headed families, two fathers were unable to participate in the interviews 
Table 3: Description of the Family Mealtime Orientations and the Corresponding Messages/Goals Parents have for Children about Mealtimes, Food and Eating

\section{Orientation}

\section{Description}

Parents described the focus of the family meal is to bring the family together and to connect socially. Parents discussed how social interaction was a more important aspect of the

Meals for

Togetherness $(n=7)$

Meals for Nutrition

Messaging $(\mathbf{n}=8)$ to talk about the importance of culture in their mealtime routine and food choices.

Parents described that the focus of the family meal is to support healthful eating.

Parents discussed how eating together at mealtimes was driven by a desire to ensure kids are eating well and the importance of educating their child about nutrition and portion sizes.

For some families, this
Representative Quotes Describing the

Mealtime Orientation

"I think everyone does completely different things, but meals are a priority for us. We like food and we like being together." (Family 1, Mother)

"Having good conversation is more important than the actual food." (Family 12, Mother)

"...mealtimes are fun, an enjoyable process, it's a social process.” (Family 20, Father) desire for healthy meals was driven by concern or uncertainty towards what their child should be eating and for others it stemmed from body image challenges and intergenerational body shame.

"I mean I definitely think about [nutrition] a lot, I would say. [...] we have to feed him good food. He won't necessarily choose the best food. But as long as he's here and we're feeding him, he's fine" (Family 9, Mother)

"Every day that's one of [my son's] primary objectives, is to eat healthy." (Family 13, Father) from my mom, who actually got mad at me when I told her I had no intention of losing weight for the wedding. Because I had to, I had to. And then it became 16, Mother) I'm not sure how many are founded, but,

\section{Messages and goals for}

"Ummm so, and a lot of that was coming she thought that was, she just thought that something that I just fixated on." (Family

Umm, I mean, like as a first-time parent I feel like I have lots of concerns. (laughs) umm, I am concerned about how much umm like grain products she eats. (Family 14, Mother) children about mealtimes,

food and eating

The main goal of mealtimes is to share messages about food being a time to connect socially and to bond as a family. Parents did not have specific nutrition related goals for eating together, but instead wanted their child to love eating and cooking out of pure enjoyment.

These parents reported very few memories of receiving specific nutrition messaging or food battles which is consistent with their current mealtime

environment

Representative Quotes of the

messages and goals for children

"We always make an effort to like not have TV on, um, to sit at the table, uh, to talk to each other. We explain to [target child] why we do that, 'cause it's the only time that we all get to see each other. So, um, yeah, I would definitely say that we make a point to have it, dinner, be dinner." (Family 10, Father)

"I want them to enjoy it. Like, I do. You know, I like eating. Yeah, and I want [mealtimes] to be a joy for them." (Family 4, Mother)

"Yeah, I think that we've also been hammering in like, not, overtly, but, it is just because of the way we do stuff, umm, portion control. [...] So, I think she's got great grasp of what uhh, what she should eat a lot of, and what she should not eat a lot of." (Family 18, Father)

The main mealtime goal was about ensuring their children have a healthy diet. These parents did not report specific goals surrounding togetherness. Many parents talked about wanting to control their child's intake.

"I wanna make sure that he doesn't get too into like carb heavy stuff." (Family 16, Mother)

"I would be hopeful that [my children] one day would be like 'I'm going to make Spaghetti Carbanara!' I'd be like 'That's okay, but it's full of fat'." (Family 2, Mother)

"I think just, food is going to make your body grow and it's going to give you energy to do the things that you want, and it will make you feel less crappy (laughs)." (Family 19, Mother) 


\section{Orientation}

\section{Description}

Parents with this onily meals

as being very functional and

as a required part of

parenting. Parents described

how meals serve as a time to

accomplish the necessary

goal of feeding their

children.

$(\mathbf{n}=5)$

\section{Representative Quotes Describing the \\ Mealtime Orientation} think we really have, like a, I don't think
"The meals for us, it's mostly about getting fed." (Family 7, Mother)

"As for like, the table routine, I don't we have like a specific [routine], no.

Yeah, but just mostly hurry up, get home, eat and then we're done." (Family 8, Mother)

So yeah, [our meals are] just come up and, you know, sit down in front of the [TV], give him ten minutes and he'll just sit there and eat, and [then] he'll be back on the floor playing or wanting to go outside." (Family 11, Father)
Messages and goals for

children about mealtimes,

food and eating

The main mealtime goal for

families in the 'Meals for

Necessity' orientation was to

accomplish the act of feeding

themselves and their children

because eating is a necessity.

While parents with this

orientation did not have goals of togetherness, they did try to

make meals a priority. They

mentioned having some

nutritional goals, which

demonstrates how orientations

overlap. However, such goals

seemed to be aspirational, with

parents often using words such as "hope".
Representative Quotes of the

messages and goals for children

"We try with the kids, well, actually, al the time we try and make it a habit for them, um, three meals a day." (Family 8, Mother)

"It shouldn't be work, you know, it shouldn't be a burden to make food and eat it." (Family 17, Mother)

"I have gotten her used to watching TV and having dinner, because it's quiet time for me, I can just eat and be like 'nourish myself, turn off my brain'." (Family 7, Mother) 